\title{
Subject index
}

\author{
Volume 9 (1997)
}

Acquired brain injury; Educational planning; Educational strategies; Student outcomes 9, 133

Acute disseminated encephalomyelitis; Acute inflammatory demyelinating polyneuropathy; Rehabilitation 9, 237

Acute inflammatory demyelinating polyneuropathy; Acute disseminated encephalomyelitis; Rehabilitation 9, 237

Agitation; Anosognosia; Environmental modifications; Outcome 9,221

Anosognosia; Agitation; Environmental modifications; Outcome 9, 221

Aphasia; Speech production; Left vs. right hemisphere 9, 89

Apraxia; Praxis; Right hemisphere; Skilled movements 9, 45

Behavior; Stroke; Rehabilitation; Therapy; Upper extremity; Motor control 9, 29

Behavioral arousal; Sensory stimulation; Comatose; Tetraplegic 9, 227

Bilateral deficits; Kinematic analysis; Fitts tapping task 9, 71

Brain injury information; Pediatrics; Families; Educators; Clinicians; Internet 9, 149

Brain injury; Traumatic; Strategies; People 9, 205

CNS; Neural regeneration; Spinal cord; Neural grafting $\mathbf{9}$, 187

Cerebrovascular accident; Motor performance; Muscle activation; Weakness 9, 3

Children; Families; Traumatic brain injury; Practical strategies 9,159
Children; Head injury; Traumatic brain injury; Clinical pathways 9,123

Clinical pathways; Children; Head injury; Traumatic brain injury 9,123

Clinicians; Brain injury information; Pediatrics; Families; Educators; Internet 9, 149

Cognistat (NCSE); Elderly; Cognitive assessment; Dementia; Neurosurgical 9, 179

Cognitive assessment; Cognistat (NCSE); Elderly; Dementia; Neurosurgical 9, 179

Comatose; Sensory stimulation; Behavioral arousal; Tetraplegic 9, 227

Deglutition disorders; Dysphagia; Long-term follow-up; Swallowing disability; Swallowing therapy; Deglutition 9, 195

Deglutition; Dysphagia; Long-term follow-up; Swallowing disability; Swallowing therapy; Deglutition disorders 9, 195

Dementia; Cognitive assessment; Cognistat (NCSE); Elderly; Neurosurgical 9, 179

Development; Pediatric traumatic brain injury; Rehabilitation; Transitions 9, 105

Dysphagia; Long-term follow-up; Swallowing disability; Swallowing therapy; Deglutition; Deglutition disorders 9, 195

Educational strategies; Acquired brain injury; Educational planning; Student outcomes 9, 133

Educational planning; Acquired brain injury; Educational strategies; Student outcomes 9, 133 
Educators; Brain injury information; Pediatrics; Families; Clinicians; Internet 9, 149

Elderly; Dementia; Cognitive assessment; Cognistat (NCSE); Neurosurgical 9, 179

Emergency services; Pediatric; Traumatic brain injury; Trauma services; Rehabilitation services 9, 113

Environmental modifications; Anosognosia; Agitation; Outcome 9, 221

Epidemiology; Violence-related injuries; Prevention 9, 167

Families; Brain injury information; Pediatrics; Educators; Clinicians; Internet 9, 149

Families; Children; Traumatic brain injury; Practical strategies 9, 159

Fitts tapping task; Kinematic analysis; Bilateral deficits 9, 71

Head injury; Children; Traumatic brain injury; Clinical pathways 9,123

Internet; Brain injury information; Pediatrics; Families; Educators; Clinicians 9, 149

Kinematic analysis; Bilateral deficits; Fitts tapping task 9, 71

Left vs. right hemisphere; Speech production; Aphasia 9, 89

Long-term follow-up; Dysphagia; Swallowing disability; Swallowing therapy; Deglutition; Deglutition disorders 9, 195

Motor control; Stroke; Rehabilitation 9, 57

Motor control; Stroke; Rehabilitation; Therapy; Upper extremity; Behavior 9, 29

Motor performance; Muscle activation; Cerebrovascular accident; Weakness 9,3

Muscle activation; Motor performance; Cerebrovascular accident; Weakness 9,3

Neural grafting; Neural regeneration; CNS; Spinal cord 9, 187

Neural regeneration; CNS; Spinal cord; Neural grafting 9, 187

Neurosurgical; Cognitive assessment; Cognistat (NCSE); Elderly; Dementia 9, 179
Outcome; Anosognosia; Agitation; Environmental modifications 9, 221

Pediatric traumatic brain injury; Rehabilitation; Transitions; Development 9, 105

Pediatric; Traumatic brain injury; Emergency services; Trauma services; Rehabilitation services 9, 113

Pediatrics; Brain injury information; Families; Educators; Clinicians; Internet 9, 149

People; Brain injury; Traumatic; Strategies 9, 205

Practical strategies; Families; Children; Traumatic brain injury 9, 159

Praxis; Apraxia; Right hemisphere; Skilled movements 9, 45

Prevention; Violence-related injuries; Epidemiology 9, 167

Reflex sympathetic dystrophy; Vocational rehabilitation; Return-to-work 9, 213

Rehabilitation services; Pediatric; Traumatic brain injury; Emergency services; Trauma services 9, 113

Rehabilitation; Acute inflammatory demyelinating polyneuropathy; Acute disseminated encephalomyelitis 9, 237

Rehabilitation; Pediatric traumatic brain injury; Transitions; Development 9, 105

Rehabilitation; Stroke; Motor control 9, 57

Rehabilitation; Stroke; Therapy; Upper extremity; Motor control; Behavior 9, 29

Resistive exercise; Stroke; Spasticity 9, 17

Return-to-work; Reflex sympathetic dystrophy; Vocational rehabilitation 9,213

Right hemisphere; Apraxia; Praxis; Skilled movements 9, 45

Sensory stimulation; Behavioral arousal; Comatose; Tetraplegic 9, 227

Skilled movements; Apraxia; Praxis; Right hemisphere 9, 45 Spasticity; Stroke; Resistive exercise 9,17

Speech production; Left vs. right hemisphere; Aphasia 9, 89 
Spinal cord; Neural regeneration; CNS; Neural grafting 9, 187

Strategies; Brain injury; Traumatic; People 9, 205

Stroke; Motor control; Rehabilitation 9, 57

Stroke; Rehabilitation; Therapy; Upper extremity; Motor control; Behavior 9, 29

Stroke; Spasticity; Resistive exercise 9, 17

Student outcomes; Acquired brain injury; Educational planning; Educational strategies 9, 133

Swallowing disability; Dysphagia; Long-term follow-up; Swallowing therapy; Deglutition; Deglutition disorders 9, 195

Swallowing therapy; Dysphagia; Long-term follow-up; Swallowing disability; Deglutition; Deglutition disorders 9, 195

Tetraplegic; Sensory stimulation; Behavioral arousal; Comatose 9, 227

Therapy; Stroke; Rehabilitation; Upper extremity; Motor control; Behavior 9, 29
Transitions; Pediatric traumatic brain injury; Rehabilitation; Development 9, 105

Trauma services; Pediatric; Traumatic brain injury; Emergency services; Rehabilitation services 9, 113

Traumatic brain injury; Children; Head injury; Clinical pathways 9,123

Traumatic brain injury; Families; Children; Practical strategies 9,159

Traumatic brain injury; Pediatric; Emergency services; Trauma services; Rehabilitation services 9,113

Traumatic; Brain injury; Strategies; People 9, 205

Upper extremity; Stroke; Rehabilitation; Therapy; Motor control; Behavior 9, 29

Violence-related injuries; Prevention; Epidemiology 9, 167

Vocational rehabilitation; Reflex sympathetic dystrophy; Return-to-work 9, 213

Weakness; Motor performance; Muscle activation; Cerebrovascular accident $\mathbf{9 , 3}$ 